\title{
Asymmetric Dichoptic Masking in Visual Cortex of Amblyopic Macaque Monkeys
}

\author{
Christopher Shooner, Luke E. Hallum, -Romesh D. Kumbhani, Virginia García-Marín, Jenna G. Kelly, \\ Najib J. Majaj, J. Anthony Movshon, and Lynne Kiorpes \\ Center for Neural Science, New York University, New York, New York 10003
}

In amblyopia, abnormal visual experience leads to an extreme form of eye dominance, in which vision through the nondominant eye is degraded. A key aspect of this disorder is perceptual suppression: the image seen by the stronger eye often dominates during binocular viewing, blocking the image of the weaker eye from reaching awareness. Interocular suppression is the focus of ongoing work aimed at understanding and treating amblyopia, yet its physiological basis remains unknown. We measured binocular interactions in visual cortex of anesthetized amblyopic monkeys (female Macaca nemestrina), using 96-channel "Utah" arrays to record from populations of neurons in V1 and V2. In an experiment reported recently (Hallum et al., 2017), we found that reduced excitatory input from the amblyopic eye (AE) revealed a form of balanced binocular suppression that is unaltered in amblyopia. Here, we report on the modulation of the gain of excitatory signals from the AE by signals from its dominant fellow eye (FE). Using a dichoptic masking technique, we found that $\mathrm{AE}$ responses to grating stimuli were attenuated by the presentation of a noise mask to the $\mathrm{FE}$, as in a normal control animal. Responses to $\mathrm{FE}$ stimuli, by contrast, could not be masked from the AE. We conclude that a weakened ability of the amblyopic eye to modulate cortical response gain creates an imbalance of suppression that favors the dominant eye.

Key words: anisometropia; binocular interaction; dichoptic masking; macaque monkey; strabismus; visual cortex

Significance Statement

In amblyopia, vision in one eye is impaired as a result of abnormal early visual experience. Behavioral observations in humans with amblyopia suggest that much of their visual loss is due to active suppression of their amblyopic eye. Here we describe experiments in which we studied binocular interactions in macaques with experimentally induced amblyopia. In normal monkeys, the gain of neuronal response to stimulation of one eye is modulated by contrast in the other eye, but in monkeys with amblyopia the balance of gain modulation is altered so that the weaker, amblyopic eye has little effect while the stronger fellow eye has a strong effect. This asymmetric suppression may be a key component of the perceptual losses in amblyopia.

\section{Introduction}

Amblyopia is a disorder of development resulting from abnormal binocular visual experience. Incorrect eye alignment (strabismus) or an interocular difference in refractive power (anisometropia) can cause one eye to become dominant, while vision through the nondominant eye develops abnormally. This results

Received June 23, 2017; revised July 23, 2017; accepted July 24, 2017.

Author contributions: C.S., L.E.H., R.D.K., N.J.M., J.A.M., and L.K. designed research; C.S., L.E.H., R.D.K., V.G.-M., J.G.K., and N.J.M. performed research; C.S., V.G.-M., J.G.K., and J.A.M. analyzed data; C.S., L.E.H., J.A.M., and L.K. wrote the paper.

This research was supported by National Institutes of Health Grants EY05864 to L.K. and EY22428 to J.A.M., and National Center for Research Resources Grant RR00166 to the Washington National Primate Research Center. We thank Michael Gorman for assistance rearing and behaviorally testing animals, and Corey Ziemba for help during experiments.

Correspondence should be addressed to J. Anthony Movshon, Center for Neural Science, New York University, 4 Washington Place, \#809, New York, NY 10003. E-mail: movshon@nyu.edu.

DOI:10.1523/JNEUROSCI.1760-17.2017

Copyright $\odot 2017$ the authors $\quad 0270-6474 / 17 / 378734-08 \$ 15.00 / 0$ in poor vision through the weak, or amblyopic, eye under monocular conditions. Additionally, during normal binocular viewing, information from the amblyopic eye is suppressed, while the stronger eye dominates perception (Hess et al., 2014). Interocular suppression correlates with the severity of monocular deficits across individuals (Li et al., 2011) and may be the primary factor limiting normal development of amblyopic eye function (Sireteanu and Fronius, 1981). Clinical therapies that aim to manipulate interocular suppression have shown promise in treating amblyopia in adults, suggesting that binocular neural pathways remain modifiable into adulthood (Hess et al., 2010; Li et al., 2013; Ding and Levi, 2014).

Recent findings suggest that amblyopic suppression involves an imbalance in an early binocular gain control mechanism, which normally facilitates binocular combination through mutual inhibition between the eyes. In amblyopes, dichoptic masking is asymmetric: masking of the dominant eye by the amblyopic eye is weak compared with masking in the opposite direction 
(Harrad and Hess, 1992; Baker et al., 2008). The results of the study by Baker et al. (2008) suggest that an early attenuation of the signal from the amblyopic eye, together with normally functioning interocular gain control, creates this imbalance favoring the dominant eye. Studies of suprathreshold binocular combination also suggest a link between binocular gain control and amblyopic suppression. Dichoptic gratings presented with mismatched contrast or phase evoke a binocular percept biased toward the image of the dominant eye (Huang et al., 2009, 2011; Ding et al., 2013). This asymmetry can be explained by a disrupted gain control, in which the ability of the amblyopic eye to modulate the dominant eye is weakened. Thus, amblyopia might not involve abnormal suppression. Rather, a weakening of the influence of the amblyopic eye tips the balance of mutual inhibition in favor of the dominant eye.

In primary visual cortex (V1) of experimentally amblyopic monkeys, reduced binocular convergence leads to a larger proportion of monocular neurons (Kiorpes et al., 1998; Kiorpes and Movshon, 2004; Shooner et al., 2015) and reduced neuronal sensitivity to retinal disparity (Smith et al., 1997; Bi et al., 2011). Amblyopic V1 also contains more neurons exhibiting purely suppressive binocular interactions (Sengpiel et al., 1994; Smith et al., 1997; Kumagami et al., 2000; Mori et al., 2002; Bi et al., 2011). These cells respond to monocular stimulation only through one eye, but this response is suppressed by stimulation of the other. This "silent" suppression is observed when excitatory convergence is absent, raising the question of whether suppression itself is abnormal or a normally functioning suppressive mechanism is revealed by the absence of excitation.

Little is known of how interocular suppression shapes the responses of neurons that receive excitatory inputs from both eyes. The behavioral and modeling results discussed above suggest that amblyopic eye signals might be more susceptible to interocular gain modulation than signals from the fellow eye, but this prediction has not been tested. We therefore revisited the question of amblyopic suppression in experimentally amblyopic macaque monkeys. We have characterized the binocular receptive fields of neurons in early visual cortex (Hallum et al., 2017). Using a novel method that isolated the excitatory and suppressive contributions of each eye, we found that a large number of seemingly monocular multiunit sites were suppressed by stimulation of the amblyopic eye. This form of binocular suppression was unaltered in amblyopes but was more apparent in the presence of reduced excitation. Here, in the same neural populations, we measured interocular modulation of excitatory signals. The responses of the amblyopic eye were attenuated by a noise mask presented to the fellow eye, while fellow eye responses were immune to interocular masking by the amblyopic eye. This asymmetric modulation was independent of the directly suppressive effects described by Hallum et al. (2017) and represents a form of abnormal binocular combination not previously observed in amblyopia.

\section{Materials and Methods}

Methods for behavioral testing and physiological recordings are described in Shooner et al. (2015). We studied five amblyopic macaque monkeys (female Macaca nemestrina) and one normal female control. Amblyopia was caused in two monkeys by experimentally induced strabismus (esotropia); three others were raised with unilateral blur as a model of anisometropia. We measured contrast sensitivity as a function of spatial frequency, monocularly in both eyes of amblyopic animals and binocularly in the control animal, and fit each sensitivity function with a descriptive model. The relative difference in sensitivity between the two eyes across all spatial frequencies was quantified using fitted curves and served as a measure of the depth of amblyopia. For each animal, an amblyopia index (AI; Kiorpes et al., 1998) value was computed, which could vary between 0 (no visual deficit) and 1 (total blindness in the amblyopic eye). We ranked subjects by AI and labeled them accordingly, as follows: subject 1 had the least severe visual deficit $(\mathrm{AI}=0.21)$, while subject 6 showed severe amblyopia $(\mathrm{AI}=0.91)$. These labels were defined in a previous publication (Shooner et al., 2015), which included one amblyope not used in this study (subject 5). All animal care and experimental procedures were performed in accordance with protocols approved by the New York University Animal Welfare Committee and conformed to the National Institutes of Health Guide for the Care and Use of Laboratory Animals.

Surgical preparation and recording. Preparation for acute recording followed standard protocols described previously (Cavanaugh et al., 2002; Graf et al., 2011). Animals were anesthetized using continuous intravenous infusion of the opiate sufentanil citrate (initial dose, $6 \mu \mathrm{g} /$ $\mathrm{kg} / \mathrm{h}$ ). Muscle paralysis by infusion of vecuronium bromide (100 $\mu \mathrm{g} /$ $\mathrm{kg} / \mathrm{h}$ ) ensured the stable position of the eyes. The eyes were treated with topical atropine sulfate to dilate the pupils and were protected with gaspermeable contact lenses. We implanted a 96-electrode "Utah" array (Blackrock Systems) over the estimated border between V1 and secondary visual cortex (V2). Electrodes in the arrays were $1 \mathrm{~mm}$ long and 400 $\mu \mathrm{m}$ apart, and formed a regular, rectangular grid parallel to the cortical surface. We pneumatically inserted the array to a depth of $0.5-1.0 \mathrm{~mm}$. After the initial recording, the array was removed and either a new array or the same array was implanted at a different border location. In each animal, we performed the following two implantations in each cortical hemisphere: one medial, in cortex representing the parafoveal visual field; and one lateral, close to the cortical representation of the fovea. Raw voltage signals were bandpass filtered between 300 and $6000 \mathrm{~Hz}$ and then squared to obtain a measure of instantaneous power. The response of a multiunit cluster on a single trial was defined as the instantaneous power averaged over an $800 \mathrm{~ms}$ window aligned to stimulus onset. Figure 1 shows the time relationship between stimulus and response. Power measurements were converted to normalized units by comparison with spontaneous activity, which was measured while both eyes viewed a gray screen: deviations from baseline were divided by the magnitude of spontaneous power to convert all measurements to units of fractional increase above baseline.

Visual stimulation. We estimated refractive error and determined the direction of gaze using direct ophthalmoscopy. Appropriate lenses were used to make the retinas conjugate with stimuli presented on a gammacorrected cathode ray tube monitor (FlexScan T966, Eizo), with a spatial resolution of $1280 \times 960$ pixels, temporal resolution of $120 \mathrm{~Hz}$, and a mean luminance $35 \mathrm{~cd} / \mathrm{m}^{2}$. Viewing distance was 1.14 or $2.28 \mathrm{~m}$, at which distances the screen subtended $21^{\circ} \times 16^{\circ}$ or $10.5^{\circ} \times 8^{\circ}$. A binocular mirror system allowed independent alignment of the fovea of each eye to any point on the display. We placed the foveas so that receptive fields of neurons driven by each eye were separated by at least half the width of the screen and stimuli placed on the receptive fields of one eye were remote from those of the other eye. We generated stimuli using an Apple Macintosh computer running Expo. Stimuli were large patches of sinusoidal grating or filtered visual noise. Sixteen gratings were tested, varying in orientation $\left(0^{\circ}, 45^{\circ}, 90^{\circ}\right.$, and $\left.135^{\circ}\right)$, spatial frequency, and contrast $(15 \%$ and $30 \%)$. Two spatial frequencies were tested in each recording session and were chosen to span the range of sensitivity of the neurons tested [usually 1 and 4 cycles/degree (c/deg) for parafoveal stimuli, 2 and $8 \mathrm{c} / \mathrm{deg}$ for foveal stimuli]. Horizontal gratings drifted downward $\left(0^{\circ}\right)$, and vertical gratings drifted rightward $\left(90^{\circ}\right)$ at a rate of $2 \mathrm{~Hz}$. Noise stimuli were samples of spatiotemporal bandpass-filtered noise, created by passing a large array of Gaussian random pixel values $(512 \times 512$ pixels $\times 64$ frames) through a spatiotemporal filter, a bandpass filter in spatial frequency (Butterworth filter, $0.5-8$ or $1-16 \mathrm{c} / \mathrm{deg}$ ) and temporal frequency $(2-8 \mathrm{~Hz})$. The resulting noise samples were individually scaled to have an rms contrast of $33 \%$. All stimuli were vignetted by a large $\left(4^{\circ}\right.$ or $\left.8^{\circ}\right)$ circular aperture with smoothed edges (Cavanaugh et al., 2002). Trials were $0.8 \mathrm{~s}$ in duration and were separated by a $0.2 \mathrm{~s}$ interval. For each eye and each grating type, we measured responses to (1) monocular grating in the tested eye, (2) monocular noise in the other eye, and (3) the 
dichoptic superposition of grating and noise. Sixty repeats of each trial type were randomly interleaved. Example stimuli are shown in Figure 1.

Experimental design and statistical analyses. Amblyopes vary widely in the strength of their visual deficits, and our sample size of six subjects was necessary to span the range from mild to severe amblyopia. In each subject, we sought to maximize the number of multiunit sites characterized and found four array penetrations per animal to be an upper limit given the geometry of our placement on the V1/V2 border and the need for careful histological reconstruction of each recorded site.

Our design did not require statistical hypothesis testing or between-subject comparison. Our aim instead was to obtain a reliable estimate of effect size within each subject individually. We used a nonparametric bootstrap analysis to estimate the central tendency and dispersion of a distribution, whether of multiunit responses over many trials or of derived measures over many multiunit sites. In each case, we resampled from an empirical distribution randomly, with replacement, 100 times, computing the mean of each random sample. We report the average of these $100 \mathrm{val}-$ ues, as well as the $95 \%$ confidence interval derived from the distribution of mean estimates. Bootstrap estimates of mean spontaneous activity were additionally used to identify signif-

icantly positive responses: stimulus conditions whose mean fell significantly $(p<0.05)$ outside the distribution of mean spontaneous activity levels. Conditions in which a grating significantly increased activity above the monocular response to noise were identified in the same way, comparing mean dichoptic response to a distribution of noise-only responses.

\section{Results}

We used multielectrode arrays implanted in visual cortex to measure the strength of dichoptic masking between the eyes of normal and amblyopic monkeys. This method allowed us to measure a form of interocular suppression even when both eyes drove excitatory responses. When the response to a dichoptic stimulus was less than predicted from the sum of monocular responses, this sublinear summation revealed an interocular influence on cortical gain. In a control animal, we found that neural responses to monocular grating stimuli were reduced when broadband noise was presented simultaneously to the other eye. Figure $2 \mathrm{~A}$ plots multiunit responses recorded on 96 electrodes of one array in the control animal. A grating presented to the left eye elicited smaller responses in the dichoptic condition (vertical axis) than when presented monocularly (horizontal axis). In the reverse dichoptic condition, gratings presented to the right eye suffered similar masking from noise presented to the left eye (Fig. $2 B$ ). Note that we define the response to a grating in the presence of a mask as the change in neural activity caused by the addition of the grating, on top of any activity driven by the mask alone.

We interpret this bidirectional masking as evidence of a balanced binocular gain control mechanism, with which each eye modulates the responsivity of the other. In amblyopic subjects, this ocular balance was severely disrupted. We observed normal dichoptic masking of responses of the amblyopic eye by noise presented to the fellow eye, but no masking in the opposite direction. Figure 2, $C$ and $D$, plots data from a monkey with severe amblyopia (subject 6) in the same format as Figure 2, $A$ and $B$. Responses to gratings in the amblyopic eye were reduced in the presence of a dichoptic noise mask (Fig. 2C), but noise presented to the amblyopic eye failed to reduce the responsivity of the fellow eye (Fig. 2D), and in many cases led to response facilitation.

Grating stimuli that did not drive any monocular response often had a suppressive effect when presented in the dichoptic condition, reducing responses to the noise mask (Fig. 2, points below the origin). We characterized this form of interocular suppression using a separate experiment on the same multiunit sites, which was described in a recent publication (Hallum et al., 2017) and is discussed further below. We focus here on how suprathreshold visual responses are modulated by binocular interaction and include in our analysis only conditions in which responses to gratings were significantly positive in both the monocular and dichoptic conditions, as determined by a nonparametric bootstrap analysis (see Materials and Methods).

We measured dichoptic masking as a gain change caused by the noise mask. We computed the ratio of masked to unmasked response (Fig. 2, axes) separately for each multiunit site and each eye, and for each grating stimulus driving a significant response. This measure provides an estimate of the relative gain applied to visual signals driven by the test grating in the presence of the mask, compared with gain in the monocular condition. To summarize masking strength for a multiunit cluster, we computed the geometric mean of this gain measure across four grating orientations, two spatial frequencies, and two contrasts (15\% and 30\%).

Dichoptic gain varied among multiunit sites from $\sim 0.3$ (strong gain reduction) to 2 (gain enhancement), with a mode near 0.7 in the control animal. Figure $3 A$ plots dichoptic gain for each multiunit site recorded in the control, separately for each eye. Because the noise typically had a direct excitatory effect on neural responses in addition to its modulatory role, dichoptic gain is plotted with respect to responses to the noise, measured monocularly. In the 

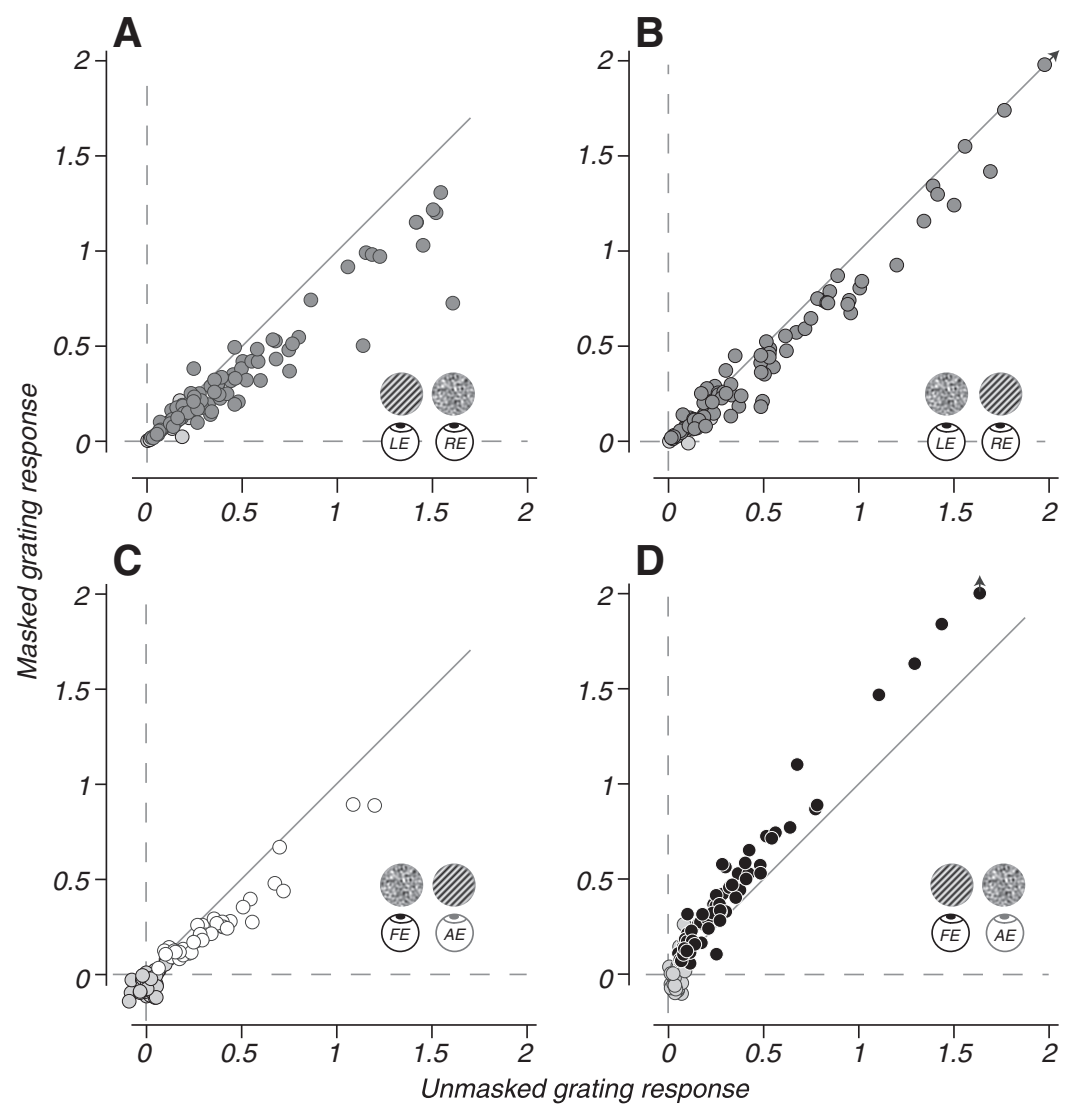

Figure 2. Multiunit responses to a grating stimulus ( $1 \mathrm{c} / \mathrm{deg}, 30 \%$ contrast) in the presence of a dichoptic noise mask are plotted with respect to monocular grating responses. Units are the fractional increase above baseline, derived by subtracting and dividing by an estimate of spontaneous activity. Responses to gratings under dichoptic conditions were derived by subtracting the response to the mask (measured monocularly) from the response to the dichoptic superposition. Purely monocular sites where the mask drove no response are shaded light gray in all panels. $A$, For an example array placement in the control animal, responses of the left eye (LE) to gratings were reduced by a mask in the right eye (RE). Each point represents the bootstrapped mean responses recorded on one electrode over 60 trials. $\boldsymbol{B}$, For the same multiunit sites, an LE mask similarly attenuated RE grating responses. $C$, In a monkey with severe amblyopia (subject 6), responses of the amblyopic eye (AE) were masked normally by noise in the fellow eye (FE). D, Grating responses in the FE were not attenuated by an AE mask, which instead caused response facilitation.

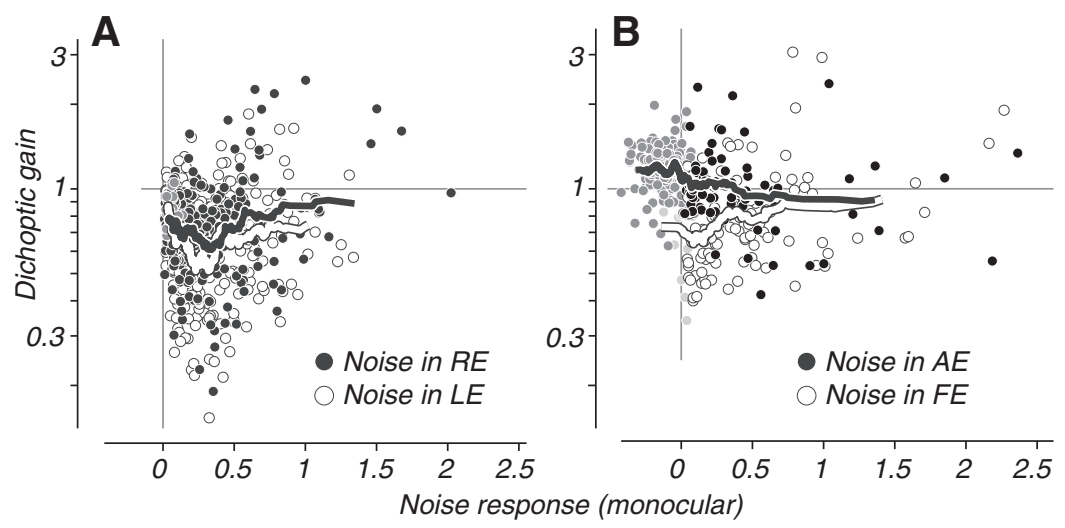

Figure 3. Relative dichoptic gain for grating stimuli is plotted with respect to monocular responses to the noise mask for every multiunit site characterized in the control and one amblyope (subject 6 ). $\boldsymbol{A}$, In the control animal, dichoptic gain values were $<1$, implying a gain reduction, even when excitatory responses to the noise were small. Running geometric means (solid lines) show that masking was of similar strength through either eye. $\boldsymbol{B}$, Noise presented to the fellow eye (FE) of an amblyope had an effect similar to that in the control (open symbols). Noise in the amblyopic eye (AE) drove fewer excitatory responses and often suppressed spontaneous activity. At sites that did respond to the mask, relative gain remained near unity, implying no modulation of fellow-eye grating responses. We focused further analysis only on sites responding significantly to the noise mask; sites not meeting this criterion are shaded in light gray. LE, Left eye; RE, right eye. control animal, the magnitude of the excitatory response to the noise did not strongly predict its effectiveness as a dichoptic mask: significant masking was observed even when the noise drove only weak responses. A running mean (Fig. 3, solid curves) shows that dichoptic masking was of similar strength through either eye over the range of observed noise-response amplitudes.

Broadband noise presented to the fellow eye of amblyopic subjects had an effect similar to that observed in the control, both in driving excitatory responses and modulating the responsivity of the amblyopic eye. Figure $3 B$ plots data from a monkey with severe amblyopia (subject 6) in the same format as in Figure $3 A$, with open symbols representing this dichoptic condition. In contrast, noise presented alone to the amblyopic eye (Fig. 3B, filled symbols) drove excitation at fewer multiunit sites, and in many cases it caused a suppression of spontaneous activity. This pattern of monocular responses is consistent with previous measurements made from the same neural population (Shooner et al., 2015; Hallum et al., 2017). In the dichoptic condition, noise in the amblyopic eye failed to reduce the responsivity of the fellow eye, leading to dichoptic gain values of $\geq 1$. This was true when the monocular responses to noise were suppressive, but also when noise drove excitatory responses in the range typical of the control animal $(\sim 0$ to 0.5$)$.

To summarize the masking effects across many multiunit sites in each animal, we consider only those cases where the noise stimulus drove at least a minimal excitatory response when presented in isolation (e.g., excluding the gray points in Fig. 3B, based on the bootstrap procedure described in Materials and Methods). This conservative choice underestimates the total difference between the eyes but allows comparison of the modulatory effect of a dichoptic mask in either eye, while approximately controlling for its direct excitatory or suppressive effect.

We observed a large asymmetry in dichoptic masking in the three most severely amblyopic subjects tested (two anisometropic, one strabismic). Figure 4 plots the distributions of dichoptic gain for each animal, separately for each eye. Figure 5 plots the geometric means of these distributions with respect to a behaviorally measured AI. In all subjects, noise presented to the fellow eye reduced the responsivity to stimulation of the amblyopic eye by an amount similar to that observed in the control. In amblyopes, noise presented to the amblyopic eye 


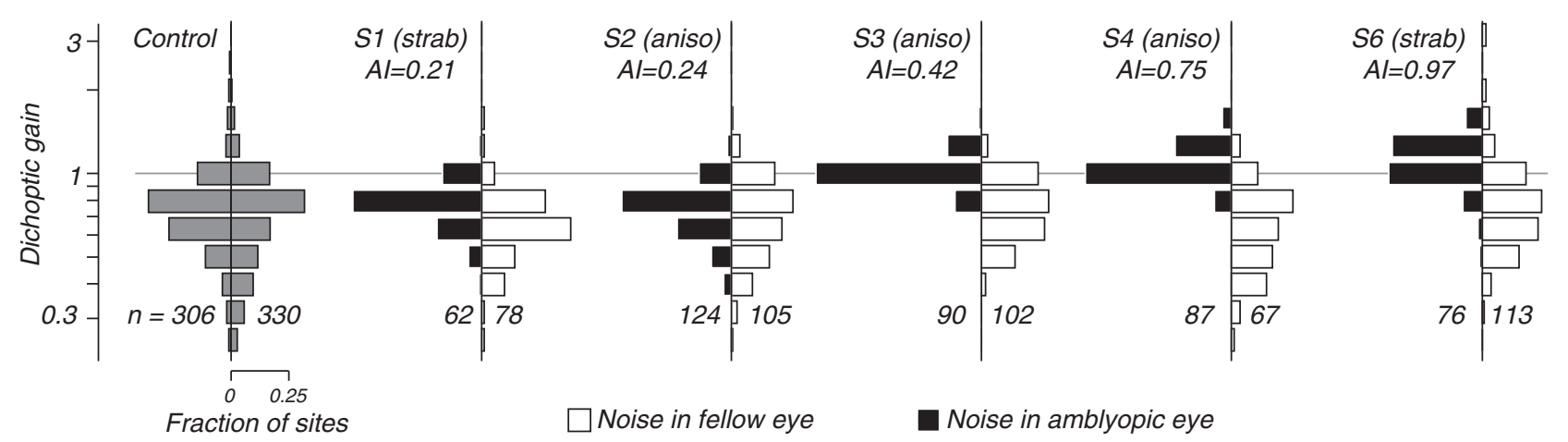

Figure 4. For each animal (ordered by severity of behavioral deficit), distributions of relative gain for grating stimuli (dichoptic/monocular) are shown separately for gratings tested in each eye. Gain was geometrically averaged across all grating types eliciting significant positive responses both monocularly and dichoptically. The control animal exhibited symmetric dichoptic masking, and values from both eyes are centered below 1 , implying gain reduction. In animals with the most severe amblyopia, masking of the responses of the amblyopic eye (AE) by noise in the fellow eye (FE) appeared normal (open bars), while distributions of dichoptic gain for the reverse dichoptic condition (filled bars) are shifted to higher values, showing a failure of the AE mask to reduce FE gain and a transition to gain enhancement. Distributions include all multiunit sites where the monocular response to noise alone was significantly positive (evaluated separately for each eye). aniso, Anisometropia; strab, strabismus.

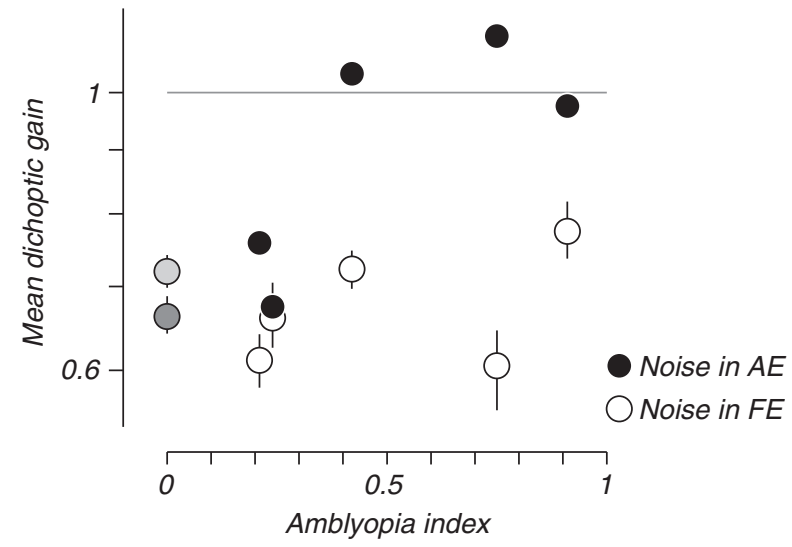

Figure 5. Dichoptic gain was geometrically averaged over multiunit sites using a bootstrap procedure. Mean gain is plotted with respect to the behaviorally derived amblyopia index (Shooner et al., 2015). Masking of the amblyopic eye (AE) by the fellow eye (FE) was normal across all subjects (open symbols), while the ability of the amblyopic eye to attenuate gain in the fellow eye (closed symbols) was reduced in the animals with more severe amblyopia.

caused no reduction in responsivity of the fellow eye (dichoptic gain, 1), and in some cases caused facilitation (gain, $>1$ ).

\section{Spatial frequency and contrast}

The imbalance in binocular gain control described above did not depend strongly on the spatial frequency or contrast of the gratings used as test stimuli. For each array recording, we chose two spatial frequencies approximately spanning the range of selectivity of the neurons tested (usually 1 and 4 or 2 and $8 \mathrm{c} / \mathrm{deg}$ ). On average, we found that responses to higher spatial frequencies suffered more dichoptic masking in both normal and amblyopic subjects. Figure 6 plots the distributions of dichoptic gain as in Figure 4 but averaged over orientation and contrast, separately for high and low spatial frequency. At both spatial frequencies tested, masking of the amblyopic eye resembled the control, while masking of the fellow eye was weaker. This is shown for subject 6 only, but we found no evidence of other frequency-dependent effects in any subject. The relative insensitivity of the amblyopic eye to the higher spatial frequency (Kiorpes et al., 1998; Shooner et al., 2015) led to fewer significant responses in this condition, preventing in-depth analysis across subjects.
Masking strength varied with the contrast of the test grating, but this effect was also small compared with the asymmetry between eyes. Figure $7, A$ and $B$, plots mean dichoptic gain with respect to the amblyopia index in the same format as in Figure 5. Figure $7 C$ directly compares masking strength between the two contrast conditions. The most common effect in both normal and amblyopic animals was weaker dichoptic masking of the lower contrast grating $(15 \%)$ than of the higher contrast grating (30\%; Fig. $7 C$, points above the diagonal). In one monkey with severe amblyopia, the opposite was true for one dichoptic condition: lower contrast gratings presented to the amblyopic eye were more strongly masked than higher contrast gratings. The masking of low contrasts in this case was also significantly stronger than that in the control (Fig. 7B).

\section{Comparison with binocular suppression}

In a separate experiment, reported in the study by Hallum et al. (2017) and described in the Discussion section, we characterized the binocular receptive field of each multiunit site using a novel method to distinguish excitatory and suppressive inputs. We found that in the control animal both eyes often contributed both excitation and suppression. In amblyopes, many sites received little or no excitatory input from the amblyopic eye, while suppressive inputs remained intact. The effect of the stimulation of the amblyopic eye on many cortical sites was therefore to suppress the responses driven by the fellow eye.

To distinguish between this form of suppression and the abnormal binocular gain control described above, we compared our dichoptic gain measurement to the excitation index (EI) defined in the study by Hallum et al. (2017). This measure, computed separately for each eye and each multiunit site, is zero when excitation and suppression are balanced, and positive or negative when one or the other dominates. Figure 8 plots this comparison for the right eye of the control animal (Fig. 8A) and the amblyopic eye of subject 6 (Fig. 8B). Most multiunit sites in the control had positive EI values: excitatory input from the right eye was accompanied by weaker suppression. The dichoptic gain measure shows that noise presented to the right eye had a normal masking effect, reducing the response to left eye stimuli.

In the amblyope, many sites had negative EI values: the amblyopic eye contributed strong suppressive input and little excitation. Surprisingly, at these sites suppressed by the amblyopic eye, noise presented to the amblyopic eye failed to mask re- 


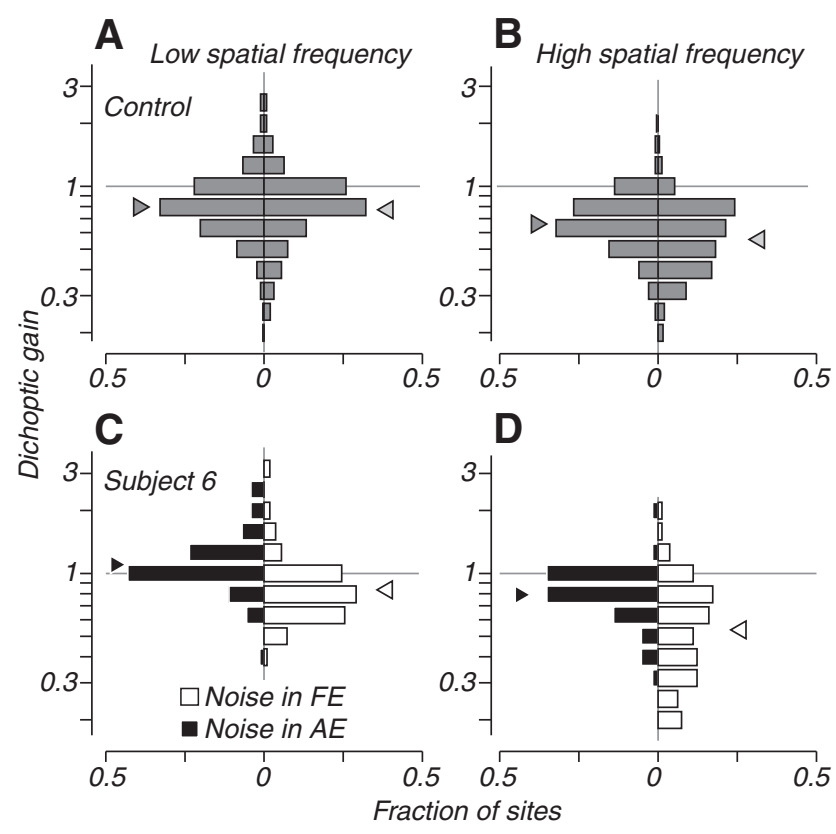

Figure 6. Responses to higher spatial frequency test gratings were more strongly masked in both the control animals and amblyopes. $A, B$, Distributions of dichoptic gain are plotted for each eye of the control animal in the same format as in Figure 4 , after averaging over orientation and contrast, separately for the two tested spatial frequencies. $\boldsymbol{C}, \boldsymbol{D}, \mathrm{As}$ in $\boldsymbol{A}$ and $\boldsymbol{B}$, but for an animal with severe amblyopia (subject 6). For both spatial frequencies, masking of the amblyopic eye (AE) appeared normal, while masking of the fellow eye (FE) was weaker. Arrows mark bootstrap estimates of the geometric mean gain.

sponses of the fellow eye, leading to dichoptic gain values of $\geq 1$. These results highlight the distinction between changes in gain modulation and changes in direct suppression that we observed in these animals. The opposite effects of amblyopia on the two kinds of dichoptic interaction suggest that they might be mediated by two distinct mechanisms. Most sites showing direct suppression were excluded from our summary of masking results, which included only cases where the mask drove a significant positive response. Sites included in this summary (Fig. 8, black symbols) showed normally balanced excitation and suppression from the amblyopic eye by the measure of the study by Hallum et al. (2017; EI >0), yet on average showed the same failure to mask fellow eye responses (Fig. 5).

\section{Discussion}

We used a dichoptic masking technique to measure interocular modulation of neural responsivity. In a normal control animal, we observed balanced, bidirectional masking: grating stimuli presented to either eye elicited smaller response increments in the presence of a dichoptic noise mask than when presented alone. In amblyopes, masking was consistently asymmetric: masking of the amblyopic eye by the fellow eye appeared normal, while the fellow eye was immune to masking by the amblyopic eye. We interpret these results in terms of a binocular gain control mechanism whereby signals from the two eyes modulate each other via mutual inhibition. A weakened contribution to such a mechanism from the amblyopic eye would create an imbalance in favor of the fellow eye, supporting its perceptual dominance during binocular viewing.

By measuring changes in gain caused by a dichoptic mask, we characterized a form of interocular suppression even at cortical sites receiving excitatory input from both eyes. At many sites, our noise stimulus elicited a significant response when presented alone, and the dichoptic addition of a test grating further increased this response. When this increase was smaller than the response elicited by the grating monocularly, we infer that the mask reduced the gain applied to signals driven by the grating. In this way, our approach differs from previous binocular experiments that have focused on "purely" suppressive effects, conditions where the addition of a dichoptic stimulus led to decreases in firing rate.

Previous studies have documented interocular suppression in visual cortex of strabismic cats (Sengpiel et al., 1994) and amblyopic monkeys (Smith et al., 1997; Kumagami et al., 2000; Mori et al., 2002; Bi et al., 2011). In these animals, most neurons lacked normal excitatory binocular convergence and disparity tuning. Many monocularly excited cells were also strongly suppressed by dichoptic stimulation of the nonpreferred eye. The authors concluded that while these suppressive effects were unusually common in amblyopes, they may reflect a normally functioning suppressive mechanism, uncovered by the absence of excitation in the amblyopic eye.

We recently reported the results of a binocular experiment (Hallum et al. (2017)), which expanded on these findings to clarify the relationship between altered excitation and binocular suppression. For the same neural populations described in this study, we characterized binocular receptive fields using a novel method that modulated the contrast seen by each eye at a different temporal frequency. This "frequency tagging" method allowed the isolation of the contribution of each eye to the summed binocular response. By also modulating contrast over space and applying a simple receptive field model, we estimated the excitatory and suppressive contribution of each eye to each multiunit site.
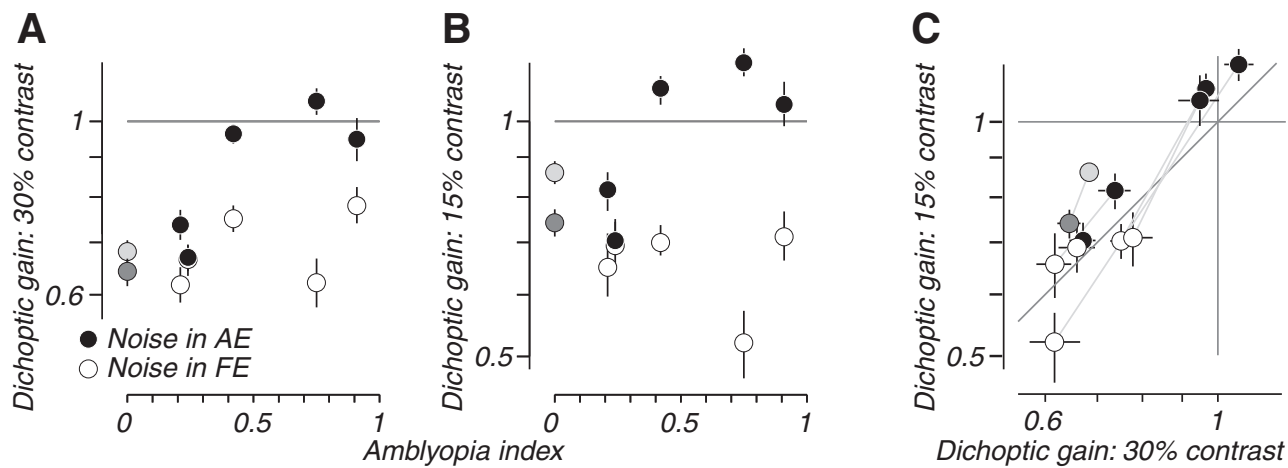

Figure 7. $\boldsymbol{A}, \boldsymbol{B}$, Mean dichoptic gain is plotted with respect to amblyopia index as in Figure 5 , separately for higher $(\boldsymbol{A})$ and lower $(\boldsymbol{B})$ contrast test gratings. $\boldsymbol{C}$, Data from $\boldsymbol{A}$ and $\boldsymbol{B}$ are replotted, comparing dichoptic gain between the two contrast conditions. Gray lines connect points representing the two eyes of the same animal. Differences between low and high contrast (deviations from the diagonal) were small compared with differences between the eyes of amblyopes (distance between connected pairs). AE, Amblyopic eye; FE, fellow eye. 
Receptive fields characterized in the control animal typically received excitatory and suppressive inputs from both eyes. In amblyopes, excitation from the amblyopic eye was reduced or absent, while binocular suppression remained intact. We conclude that this form of interocular suppression is unaltered in amblyopia, but a disrupted balance between suppression and excitation leads, in many cases, to an abnormal suppressive effect of the stimulation of the amblyopic eye.

A comparison of the current results to those of the study by Hallum et al. (2017) suggests that multiple interocular suppressive mechanisms are involved in amblyopia. Binocular suppression (Hallum et al., 2017) and interocular gain modulation were both abnormal in our amblyopic subjects but were unbalanced in opposite directions: where the direct suppressive influence of the amblyopic eye appeared abnormally strong (due to reduced excitation), its ability to mask responses of the fellow eye was abnormally weak. This was true for neural populations, on average, and also for individual multiunit sites (Fig. 8). We also observed weakened dichoptic masking at sites that, by the measure of Hallum et al. (2017), received normally balanced excitation and suppression from the amblyopic eye.

The neural circuitry supporting these interocular effects is not well understood. Sengpiel and Blakemore (1996) proposed that normal inhibitory connections between cortical ocular dominance columns may be relatively diffuse compared with more precisely wired excitatory connections. The development of excitatory pathways may then depend more critically on congruent binocular input. These connections are lost in amblyopia, while diffuse interocular inhibition is maintained. The persistence of a bidirectional inhibitory pathway may account for our finding of unaltered binocular suppression (Hallum et al., 2017), but not the observed asymmetry in dichoptic masking. Signals responsible for interocular gain changes may instead be carried by excitatory intercolumnar projections that then synapse onto local inhibitory circuits. This model is consistent with our findings that the amblyopic eye is weakened both in its ability to drive excitatory responses (Shooner et al., 2015; Hallum et al., 2017) and in its ability to modulate cortical responsivity. From a computational perspective, we might expect the summed activity of a pool of excitatory neurons to provide the relevant signal for purposes of gain control, namely, an estimate of average contrast energy seen by the amblyopic eye (Heeger, 1992; Carandini et al., 1997).

Imbalanced binocular gain control provides a possible neural basis for a variety of psychophysical findings. The studies of Harrad and Hess (1992) and Baker et al. (2008) both found asymmetric dichoptic masking in human amblyopic subjects. A pedestal mask in the dominant eye elevated amblyopic-eye thresholds more than a mask of the same absolute contrast in the reverse condition. This observation is consistent with our finding that the same noise mask can attenuate neural responses when presented to the fellow eye but not when presented to the amblyopic eye. A secondary question, which has been answered less consistently, is whether this asymmetry persists when stimuli are described on threshold-normalized axes to control for interocular differences in sensitivity. We cannot address this question directly with our current data. All stimuli in this study were suprathreshold for both eyes (with the possible exception of high-frequency, lowcontrast gratings in severe amblyopes), and our test contrasts spanned a small range ( 1 octave) compared with differences in behavioral detection thresholds (Shooner et al., 2015). An avenue for future work is to more closely replicate psychophysical experiments by systematically varying mask and test contrasts. This will allow us to determine whether a simple attenuation of signals from the amblyopic eye can account for both sensitivity loss and asymmetric masking, as proposed by Baker et al. (2008), or whether abnormal masking implies an additional deficit beyond those limiting detection (Harrad and Hess, 1992).

Studies of binocular combination in humans with amblyopia have found that the fusion of a dichoptic pair of gratings evokes a percept that is biased toward the image of the dominant eye (Huang et al., 2009, 2011, Ding et al., 2013). This asymmetry is also attributed to an imbalanced binocular gain control system, in which the amblyopic eye suffers reduced influence over signals from the fellow eye. These studies also report abnormal interocular gain enhancement (Ding et al., 2013), cases where increasing amblyopic-eye contrast paradoxically increased the perceptual dominance of the fellow eye. A transition from gain reduction to gain enhancement with amblyopia was also a prominent feature of our data (Fig. 4), providing evidence in support of models containing an active form of binocular cooperation, balanced with interocular competition (Ding et al., 2013). The fact that we observed gain enhancement when the direct effect of the noise mask was suppressive (Figs. 3, 8) argues against alternative models that account for supralinear binocular combination using an expansive nonlinearity (Meese et al., 2006; Baker et al., 2008; Moradi and Heeger, 2009).

Our findings support recent suggestions that manipulating interocular suppression might prove to be an effective amblyopia therapy (Hess et al., 2010; Li et al., 2013; Ding and Levi, 2014). Moreover, we provide the first physiological evidence that the plasticity required for restoration of normal binocular function need not include a weakening of suppression of the amblyopic eye, but rather a strengthening of the suppressive influence of the amblyopic eye over the dominant eye. The strengthening of connections that synapse onto dominant-eye neurons may require 
those postsynaptic neurons to be active, and silencing these cells by patching the dominant eye prevents the necessary synaptic changes. From this perspective, a weak but visible stimulus presented to the dominant eye might provide the necessary target signal for modulation by the amblyopic eye, while avoiding the reciprocal suppression that normally overpowers the signals of the amblyopic eye in cortex.

\section{References}

Baker DH, Meese TS, Hess RF (2008) Contrast masking in strabismic amblyopia: attenuation, noise, interocular suppression and binocular summation. Vision Res 48:1625-1640. CrossRef Medline

Bi H, Zhang B, Tao X, Harwerth RS, Smith EL 3rd, Chino YM (2011) Neuronal responses in visual area V2 (V2) of macaque monkeys with strabismic amblyopia. Cereb Cortex 21:2033-2045. CrossRef Medline

Carandini M, Heeger DJ, Movshon JA (1997) Linearity and normalization in simple cells of the macaque primary visual cortex. J Neurosci 17:86218644. Medline

Cavanaugh JR, Bair W, Movshon JA (2002) Nature and interaction of signals from the receptive field center and surround in macaque V1 neurons. J Neurophysiol 88:2530-2546. CrossRef Medline

Ding J, Levi DM (2014) Rebalancing binocular vision in amblyopia. Ophthalmic Physiol Opt 34:199-213. CrossRef Medline

Ding J, Klein SA, Levi DM (2013) Binocular combination in abnormal binocular vision. J Vis 13(2):14 1-31. CrossRef Medline

Graf AB, Kohn A, Jazayeri M, Movshon JA (2011) Decoding the activity of neuronal populations in macaque primary visual cortex. Nat Neurosci 14:239-245. CrossRef Medline

Hallum LE, Shooner C, Kumbhani RD, Kelly JG, Garcia-Marin V, Majaj NJ, Movshon JA, Kiorpes L (2017) Altered balance of receptive field excitation and suppression in visual cortex of amblyopic macaque monkeys. J Neurosci. Advance online publication. Retrieved July 31, 2017. doi: 10.1523/JNEUROSCI.0449-17.2017. CrossRef Medline

Harrad RA, Hess RF (1992) Binocular integration of contrast information in amblyopia. Vision Res 32:2135-2150. CrossRef Medline

Heeger DJ (1992) Normalization of cell responses in cat striate cortex. Vis Neurosci 9:181-197. CrossRef Medline

Hess RF, Mansouri B, Thompson B (2010) A binocular approach to treating amblyopia: antisuppression therapy. Optom Vis Sci 87:697-704. CrossRef Medline

Hess RF, Thompson B, Baker DH (2014) Binocular vision in amblyopia: structure, suppression and plasticity. Ophthalmic Physiol Opt 34:146162. CrossRef Medline
Huang CB, Zhou J, Lu ZL, Feng L, Zhou Y (2009) Binocular combination in anisometropic amblyopia. J Vis 9(3):17 1-16. CrossRef Medline

Huang CB, Zhou J, Lu ZL, Zhou Y (2011) Deficient binocular combination reveals mechanisms of anisometropic amblyopia: signal attenuation and interocular inhibition. J Vis 11(6):4 1-17. CrossRef Medline

Kiorpes L, Movshon JA (2004) Neural limitations on visual development in primates. In: The visual neurosciences (Chalupa LJ, Werner JS, eds), pp 159-173. Cambridge, MA: MIT.

Kiorpes L, Kiper DC, O’Keefe LP, Cavanaugh JR, Movshon JA (1998) Neuronal correlates of amblyopia in the visual cortex of macaque monkeys with experimental strabismus and anisometropia. J Neurosci 18:64116424. Medline

Kumagami T, Zhang B, Smith EL 3rd, Chino YM (2000) Effect of onset age of strabismus on the binocular responses of neurons in the monkey visual cortex. Invest Ophthalmol Vis Sci 41:948-954. Medline

Li J, Thompson B, Lam CSY, Deng D, Chan LYL, Maehara G, Woo G, Yu M, Hess RF (2011) The role of suppression in amblyopia. Invest Opthalmol Vis Sci 52:4169-4176. CrossRef

Li J, Thompson B, Deng D, Chan LY, Yu M, Hess RF (2013) Dichoptic training enables the adult amblyopic brain to learn. Curr Biol 23:R308 R309. CrossRef Medline

Meese TS, Georgeson MA, Baker DH (2006) Binocular contrast vision at and above threshold. J Vis 6:1224-1243. CrossRef Medline

Moradi F, Heeger DJ (2009) Inter-ocular contrast normalization in human visual cortex. J Vis 9(3):13 1-22. CrossRef Medline

Mori T, Matsuura K, Zhang B, Smith EL 3rd, Chino YM (2002) Effects of the duration of early strabismus on the binocular responses of neurons in the monkey visual cortex (V1). Invest Ophthalmol Vis Sci 43:1262-1269. Medline

Sengpiel F, Blakemore C (1994) Interocular control of neuronal responsiveness in cat visual cortex. Nature 368:847-850. CrossRef Medline

Sengpiel F, Blakemore C, Kind PC, Harrad R (1994) Interocular suppression in the visual cortex of strabismic cats. J Neurosci 14:6855-6871. Medline

Shooner C, Hallum LE, Kumbhani RD, Ziemba CM, Garcia-Marin V, Kelly JG, Majaj NJ, Movshon JA, Kiorpes L (2015) Population representation of visual information in areas V1 and V2 of amblyopic macaques. Vision Res 114:56-67. CrossRef Medline

Sireteanu R, Fronius M (1981) Naso-temporal asymmetries in human amblyopia: consequence of long-term interocular suppression. Vision Res 21:1055-1063. CrossRef Medline

Smith EL 3rd, Chino YM, Ni J, Cheng H, Crawford ML, Harwerth RS (1997) Residual binocular interactions in the striate cortex of monkeys reared with abnormal binocular vision. J Neurophysiol 78:1353-1362. Medline 